\title{
A Review on Selective Estrogen Receptor Modulators
}

\section{Himadri Bandolia, Waseem Khan}

\section{Siddhartha Institute of Pharmacy, Near IT Park, Dehradun- 248001, Uttarakhand, INDIA}

Received: 02 July,2019

Accepted: 09 Sept,2019

*Correspondence to:

Himadri Bandolia,

Email: himadri0804@gmail.com

Copyright: () the author(s), publisher and licensee Indian Academy of Pharmacists. This is an open-access article distributed under the terms of the Creative Commons Attribution Non-Commercial License, which permits unrestricted non-commercial use, distribution, and reproduction in any medium, provided the original work is properly cited.

Published by: OZZIE Publishers

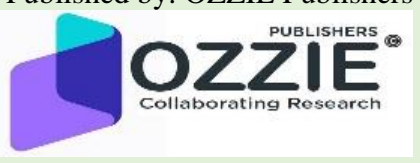

\section{Abstract}

Selective estrogen receptor modulators drugs, which exert estrogenic as well as antiestrogenic actions in a tissue selective manner, are used for the treatment of osteoporosis, breast cancer and with effects on the uterus and vagina that depend on the interaction with the estrogen receptors in target tissues. Due to decline of estrogen levels after menopause, hot flashes and sweating occurred as the part of the menopausal vasomotor symptoms with the estrogen related urogenital atrophy and loss of bone density. Selective estrogen receptor modulators (SERMs) has three compounds Tamoxifen Citrate, Raloxifene, Bazedofene. Tamoxifen acts as selective estrogen receptor modulator or as a partial agonist of the estrogen receptors. It has mixed estrogenic and anti-estrogenic activity with its profile of effects deficiency by tissue. Raloxifene is used for the treatment of oestoporosis in postmenopausal women and also reduces breast density. Bazedofene has been also developed for the treatment of osteoporosis. The estrogen receptor has two subunits alpha and beta and SERMs interact either of these subunits and form this interaction, there is a certain level of target site specificity and tissue specificity of SERMs action. Tamoxifen represented agonist effects in inhibiting neutralised migration and preventing arthritis progression in ovariectomized mice. Clomiphene citrate and tamoxifen commonly used SERMs for the induction of ovulation. that Bazedoxifen enhances OPCs into functional oligodendrocytes which enhanced OPC differentiation and remyelination. Estrogen receptor alpha play a vital role in the etiology, treatment and prevention of the majority breast cancer. The SERMs raloxifene and bazedoxifen also both reportedly inhibit bone resorptive activity in postmenopausal osteoporosis patients and have been used to prevent bone fragility fractures.

Keywords: SERMs, Osteoporosis, Menopause

\section{INTRODUCTION}

In postmenopausal women, selective estrogen receptor modulators drugs, which exert estrogenic as well as antiestrogenic actions in a tissue selective manner, are used for the treatment of osteoporosis, breast cancer and with effects on the uterus and vagina that depend on the interaction with the estrogen receptors in target tissues.[1] Menopause is the natural step of aging in which end of menstruation after the last menstrual period in the last 12 months take place.[2] Due to decline of estrogen levels after menopause, hot flashes and sweating occurred as the part of the menopausal vasomotor symptoms with the estrogen related urogenital atrophy and loss of bone density. Hormone Replacement Therapy is used for the treatment of these symptoms, but due to the presence of risk of using hormone replacement therapy causing breast cancer, endometrial cancer, thromboembolism, vaginal bleeding, which may reduce the use of hormone therapy.[3]

Selective Estrogen Receptor Modulators (SERMs) has three compounds Tamoxifen Citrate, Raloxifene, Bazedoxifene. These three compounds are used for clinical use. Tamoxifen Citrate is a first line hormonal treatment drug. It was made in 1962 by chemist Dora Richardson. It acts as selective estrogen receptor modulator or as a partial agonist of the estrogen receptors. It has mixed estrogenic and antiestrogenic activity with its profile of effects deficiency by tissue. Currently prescribed for estrogen receptor positive breast cancer. Tamoxifen Citrate has predominantly antiestrogenic effects in breasts but predominantly estrogenic effects in the uterus and liver. It also considered as for the treatment of osteoporosis. [1]

Raloxifene is second line hormonal treatment drug. It is used for the treatment of osteoporosis in postmenopausal women and reduces breast density. It is also an estrogen receptor antagonist and act as anti-estrogen on the uterus approved by the Food and Drug administration to treat osteoporosis. Bazedoxifene has been also developed for the treatment of osteoporosis.[4]

\section{Mechanism of Action}

The mechanism of action of SERMs class of compound based on their tissue selective estrogen receptor agonist or antagonist activity in their interaction with the estrogen receptor. The estrogen receptor has two subunits alpha and beta and SERMs interact either of these subunits and form this interaction, there is a certain level of target site specificity and tissue specificity of SERMs action. This differential behaviour of SERMs depends on eliciting varying signaling properties from the estrogen receptor that is tissue specific and such effects have profound physiological effects and are not dictated at the DNA level.[5] 


\section{Re-evaluation of Medical Use on The Basis of Clinical Data}

Arthritis Use (agonist activity of tamoxifen)

Arthritis is categorised as persistent synovitis and systemic inflammation which is more affected in women than men, especially during the menopause phase in which there is reduction of sex hormones, mainly estrogen. It is also known as that estrogen receptor alpha is expressed in synovial tissues which is regulated upwards in the presence of inflammation.[6] Tamoxifen represented agonist effects in inhibiting neutralized migration and preventing arthritis progression in ovariectomized mice.[7] IL-17- producing CD4 T-helper cells (Th 17 cells) are present in the inflamed joint cavity and contribute to the progression of an early inflammation to persistent chronic arthritis.[8]

\section{Comparison between tamoxifen and clomiphene citrate}

Clomiphene citrate and tamoxifen commonly used SERMs for the induction of ovulation.[9] Ovulatory dysfunction is one of the common causes of reproduction failure in sub fertile and infertile couples.[10] In 1956, clomiphene citrate has been used as the first line method of ovulation induction in couples with an ovulatory infertility. Many studies shown that approximately $80 \%$ of women ovulate using clomiphene citrate but only $40 \%$ of women achieve pregnancy.[11] Tamoxifen has also been used for the induction of ovulation. It is firstly used as an adjuvant therapy in the treatment of breast cancer but its use as an ovulatory agent was first reported in 1973.[12] It was conducted by many previous trials that the lack of conception despite evidence of ovulation may be due to anti-estrogenic effects of clomiphene citrate on the endometrium which many manifest as thin endometrium and thus tamoxifen estrogenic effects on the endometrium and cervical mucus result in higher pregnancy rates.[13]

\section{CNS Remyelination}

In multiple sclerosis, an inflammatory autoimmune disorder of the CNS, an adaptive immune response to unknown CNS antigens results in oligodendrocyte damage, loss of myelin and neurologic dysfunction.[14] A compelling remyelinating agent, bazedoxifen- EMA approved (European Medicine Agency), found that Bazedoxifen enhances OPCs into functional oligodendrocytes which enhanced OPC differentiation and remyelination.[15]

\section{For Breast Cancer Cells}

Estrogen receptor alpha play a vital roles in the etiology, treatment and prevention of the majority breast cancer.[16] Somatic mutations to ESR1 (gene for ER alpha) ligand binding domain (LBD) were identified in $25-30 \%$ of patients who previously received endocrine treatment.[17] Bazedoxifene possesses improved inhibitory potential against the Y537S and D538G ER-alpha mutants as compared to tamoxifen and has inhibitory activity in combination with CDK416 inhibitor palbociclib.[18]

\section{For Osteoporosis}

Several SERMs are being utilized clinically. The SERMs raloxifene and bazedoxifen also both reportedly inhibit bone resorptive activity in postmenopausal osteoporosis patients and have been used to prevent bone fragility fractures. Under sex hormone depleted conditions, Hif1-alpha was demonstrated to be a therapeutic target in conditions of postmenopausal and male osteoporosis. Since in postmenopausal, estrogen deficient conditions promote osteoclast activation leading to bone loss, estrogen administration is considered a means to reverse these conditions, although treatment can have adverse effects.

However, SERMs have been found to be less effective in inhibiting osteoclastic bone resorption than bisphosphonates. Testing inhibitory effects on Hif1-alpha protein in osteoclasts in vitro is useful to screen candidate anti-bone resorptive agents before animal models.[19]

\section{Adverse effects of SERMs}

Clinical usage of SERMs medication can have several side effects. Hot flashes, vomiting, vaginal bleeding, vaginal discharge, menstrual irregularities are the side effects. Increased risk of venous thromboembolism is due to estrogenic action on clotting mechanism, by consuming tamoxifen. Raloxifene consumption causes hot flushes, and leg cramps which are quite mild. The only serious concern is 3-fold increase in risk of deep vein thrombosis and pulmonary embolism. Use of tamoxifene has also been linked to higher occurrence of cataract.[5]

\section{Conclusions}

With the advanced version of SERMs, they have become suitable for treatment of osteoporosis in postmenopausal women. SERMs also used in arthritis inflammation but require caution in their use. SERMs also have their effect pregnancy rates. After 80 years of usage, side effects are more likely to be absorbed. Based on large epidemiological studies which have shown $45 \%$ reduction in the incidence of ER- positive breast cancer, tamoxifen has been approved for primary prophylaxis of breast cancer in high -risk women. Improvement in bone mass due to anti-resorptive effect, and in lipid profile are the other benefits of tamoxifen therapy. Several long-term multicentric studies have shown that raloxifene prevents bone in postmenopausal women. Mainly SERMs are used for the treatment and prevention of breast cancer and osteoporosis in postmenopausal women and expected to be a good choice of treatment.

\section{References}

1. Archer DF. The gynecologic effects of labofoxifene, an estrogen agonist/ antagonist, in post menopause women. Menopause.2011;18:6-7.

2. World Health Organization, 1996. Research on the menopause in the 1990s WHO technical Report Series 866.

3. Jhonson SR. Menopause and hormonal replacement therapy. Med clin North Am.1998:297-320.

4. Silverman SL. Sustained efficacy and safety of bazedoxifen in preventing fractures in postmenopausal women with osteoporosis: results of a 5 year, randomized, placebo- controlled study. Osteoporosis Int.2012;351-63.

5. Ki-Chan An. Selective estrogen receptor modulators. Asian Spine J. 2016;10(4):787-791.

6. Ishizuka M. Sex steroid receptors in rheumatoid arthritis. Clin Sci. 2003;10(6):293-300.

7. Silva LAS. Agonist activity of tamoxifen, a selective estrogen receptor modulator (SERM), on arthritic ovariectomized mice. Brazilian J Med Bio Res.2018;1-5. 
8. Paulissen SMJ. The role and modulation of CCR6-1 Th 17 cell population in rheumatoid arthritis. Cytokins.2015:45-53.

9. Clark JH. The agonistic-antagonistic properties of clompihene: A review. Pharmacol Ther.1982;15.

10. Balen AH. What is Polycystic ovary syndrome (PCOS)? Are national views important?. Women Human report. 2002; 17:2219-27.

11. Eden JA. The effect of clomiphene citrate on follicular phase increase in endometrial thickness and uterine. 1989; 187-90.

12. Williamson JG. The induction of ovulation by tamoxifen. J Obst Gynaecol Br Cmnwlth,.1973;844-7.

13. Hassan ZB. Comparison between tamoxifen and clomiphene citrate for inductionof ovulation syndrome. Int J Reprod Contracept Obstet Gynecol, 2015;4(5):13491352.

14. Hauser SZ. Multiple sclerosis: prospects and promise. Ann Neurol.2013;317-327.

15. Kelsey A. Rankin. Selective Estrogen Receptor Modulators Enhances CNS Remyelination Independent of Estrogen Receptors. J Neuroscience.2019;12:2184-2194.

16. Frasor J. Selective Estrogen Receptor Modulators. Cancer Research.2004;1522-1533.

17. Toy W. ESR1 ligand- binding domain mutations in hormone- resistant breast cancer. Nature Genetics.2013;1439-1445.
18. Fanning WS. The SERM/ SERD bazedoxifene disrupts ESR1 helix 12 to overcome acquired hormone acquired hormone resistance in breast cells. elife.2018;7:1-26.

19. Morita M. Selective Estrogen Receptor Modulators Suppress Hifi-alpha protein Accumulation in Mouse Osteoclasts. PloS One. 2016;1(11):1-13.

20. Grace Y Stoles. Measuring selective estrogen receptor modulator (SERM)- Membrane interactions with second harmonic generation. J Am Chemical Society.2014;14091417.

21. Smith. Breast related effects of selective estrogen receptor modulators and tissue selective estrogen compexes. Breast Cancer Res.2014;16(3):1-15.

22. Lo Yu-Chen. Pocket similarity identifies selective estrogen receptor modulators as microtubule modulators at the taxeme site. Nature communication. 2019:10;1033.

23. Jordan VC. Selective Estrogen Receptor Modulation and Reduction in risk of Breast cancer, osteoporosis, and Coronary Heart Disease. Journal of the National Cancer Institute.2001;93(19):1449-1457.

24. Bazaid Ali. Selective Estrogen Receptor Modulators (SERM): A new choice foe postmenopausal women and physicians who worry on cancer. Med J Indonesia.2001;10(3):135-200.

25. Kenneth D.R. Setchell. Soy Isoflavones-Benefits and Risks from Nature Selective Estrogen Receptor Modulators (SERMs). J Am College of Nutrition. 2001;20(5):354s-362s. 